\title{
On the Duality of MIMO MAC and BC with AF Relays
}

\author{
Krishna S. Gomadam, Syed A. Jafar \\ Electrical Engineering and Computer Science \\ University of California, Irvine, CA 92697-2625 \\ Email: \{kgomadam, syed\}@uci.edu
}

\begin{abstract}
In this work, we consider a two-hop multiuser amplify-and-forward relay network with MIMO nodes. The results are three-fold. First, for any relay amplification matrix $D$ in the multiple-access channel (MAC), we show that duality holds when $\kappa \mathbf{D}^{\dagger}$ is employed in the broadcast channel (BC), and vice versa, where $\kappa$ is obtained from switching the total source and relay power constraints. Second, under a total network power constraint, we show that MAC-BC duality holds when $\mathrm{D}$ and $\mathrm{D}^{\dagger}$ are the relaying matrices in the $\mathrm{MAC}$ and $\mathrm{BC}$ respectively. Third, for any $\mathbf{D}$ in the MAC and $c \mathbf{D}^{\dagger}$ in the $\mathrm{BC}$ where $c$ is any positive real scalar, duality under total network power constraint holds only for the above two cases.
\end{abstract}

\section{INTRODUCTION}

The use of relaying techniques to transfer information via multiple hops and routes offers significant benefits for wireless networks that include throughput enhancement, range extension and power reduction. These benefits make cooperation and relaying a promising candidate for the next generation wireless systems. Various forwarding strategies at the relays have been studied. Among them, memoryless schemes such as amplify and forward (AF) are attractive for their simplicity and the ability to provide soft information.

In this work we consider multiuser MIMO AF relay networks. The most basic multiuser scenarios are the multiple access channel and the broadcast channel. For the singlehop communication without relays, the MAC-BC duality [1], [2], which states that the capacity region of a MAC is equal to the capacity region of the reciprocal $\mathrm{BC}$ for the same total transmit power, is well known. For AF relay networks with single antenna nodes, the following MAC-BC duality is recently shown in [3]. Consider a MAC with total user power $P$ and total relay power $P_{R}$. This MAC is dual to the $\mathrm{BC}$ obtained by reversing the direction of communication and swapping the sum relay power and total source power. For multihop networks with more than two hops, duality holds when the powers are shifted such that each hop gets the same transmit power in both the original and the dual channels. Such a duality relationship may be helpful in solving network optimization problems. For example, in [4], a three hop AF relay network is optimized using the duality relationship in [3].

Another version of duality in AF relay networks is found in [5]. Here the authors consider a multiuser AF relay network with memory where the relays can transmit a linear combination of previously received signals. For this model, the authors show an interesting duality relationship under a total network power constraint. It is shown that duality holds when the same relaying matrix is used in both the MAC and $\mathrm{BC}$ without any scaling factor. Duality under total network power constraint holds for the result in [3] as well. Thus MAC-BC duality for single antenna nodes, subject to the network power constraint, holds for two cases. Two important questions arise:

- Does duality hold for any scaled version of $\mathbf{D}^{\dagger}$ in the dual channel (with $\mathbf{D}$ in the original channel)?

- Do these duality relationships hold when the nodes have multiple antennas?

These are the questions that we answer in this paper. In the following, we consider a SIMO relay network and its dual channel. Here we highlight the differences with respect to a single antenna relay network that make it hard to establish a duality relationship.

Consider a SIMO relay network as shown in Fig. 1 where a single antenna source communicates to an $M$-antenna destination through a bunch of parallel relays. The relays may have multiple antennas and let $N$ be the total number of relaying antennas. In the first slot, the source transmits $x$ while the relays receive $\mathbf{r}=\mathbf{g} x+\mathbf{n}_{\mathbf{R}}$ where $\mathbf{g}$ represents the $N \times 1$ source-relay channel. The components of $\mathbf{n}_{\mathbf{R}}$ are $\mathcal{C N}(0,1)$ additive white Gaussian noise (AWGN). In the second slot, the relays collectively transmit $\mathbf{D r}$ where $\mathbf{D}$ is the relay amplification matrix. Note that $\mathbf{D}$ in general is a block diagonal matrix. The received signal vector at the destination is

$$
\mathbf{y}=\mathbf{H}^{\dagger} \mathbf{D g} x+\mathbf{H}^{\dagger} \mathbf{D} \mathbf{n}_{\mathbf{R}}+\mathbf{n}
$$

where $\mathbf{H}^{\dagger}$ is the $M \times N$ relay-destination channel and the components of $\mathbf{n}$ are $\mathcal{C N}(0,1)$ AWGN. In normalized-form, the input-output relationship of the relay network is

$$
\mathbf{y}^{\prime}=\left(\mathbf{H}^{\dagger} \mathbf{D} \mathbf{D}^{\dagger} \mathbf{H}+\mathbf{I}\right)^{\frac{-1}{2}} \mathbf{H}^{\dagger} \mathbf{D g} x+\mathbf{n} .
$$

Now consider a MISO relay network obtained by reversing the communication direction. Here the multiple antenna source communicates to the single antenna destination through the relays that use $\kappa \mathbf{D}^{\dagger}$ as their relaying matrix. Here $\kappa$ may denote the scaling factor used in the dual channel similar to those in [3], [5]. The received signal at the destination can be expressed as

$$
y=\kappa \mathbf{g}^{\dagger} \mathbf{D}^{\dagger} \mathbf{H} \mathbf{x}+\kappa \mathbf{g}^{\dagger} \mathbf{D}^{\dagger} \mathbf{n}_{\mathbf{R}}+n
$$

where $n$ is unit variance AWGN. In normalized form, the 


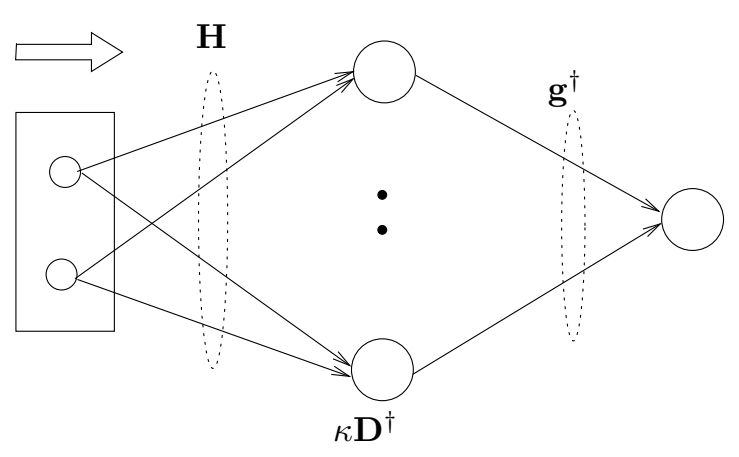

(i)

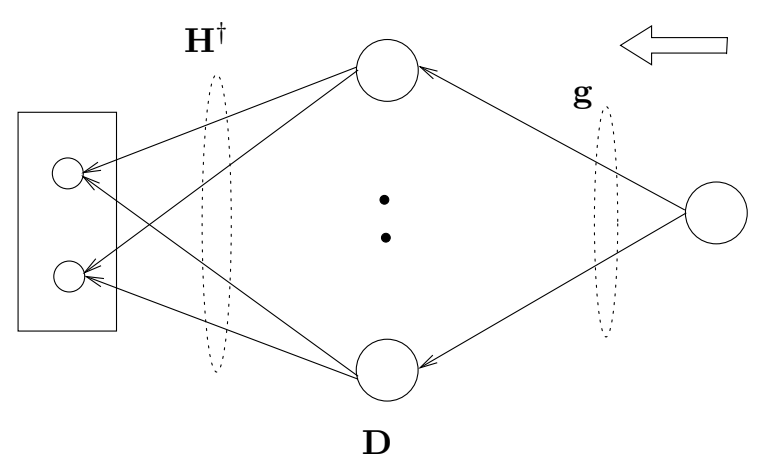

(ii)

Fig. 1. Two-hop SIMO and MISO relay networks

input-output relationship is

$$
y^{\prime}=\frac{\kappa \mathbf{g}^{\dagger} \mathbf{D}^{\dagger} \mathbf{H}}{\sqrt{1+\kappa^{2}\left\|\mathbf{g}^{\dagger} \mathbf{D}^{\dagger}\right\|^{2}}} \mathbf{x}+n .
$$

Notice that (2) and (4) have different effective channel directions. Compare these channels with the point to point SIMO and MISO channels,

$$
y=\mathbf{h}^{\dagger} \mathbf{x}+n \quad \mathbf{y}=\mathbf{h} x+\mathbf{n}
$$

that have essentially the same spatial direction. To establish duality, it is not clear whether we need a different relaying matrix for the dual channel instead of the scaled (and conjugatetransposed) versions of the original relaying matrix.

\section{DUALITY IN SIMO AND MISO RELAY NETWORKS}

Theorem 1: The capacity of a single user SIMO relay network is unchanged when the role of the transmitter and the receiver is reversed while the power constraint at the source and the relays are switched in the dual network.

Proof: To prove it, we need to show that the dual channel is at least as capable as the original channel and vice versa.

\section{A. Part 1 (MISO $\geq S I M O)$}

The input signal in the SIMO relay network is a scalar $x$ that satisfies the power constraint $P$ while the relay amplification factor D satisfies the relay power constraint. For this model (given by (1)), the capacity optimal strategy is to use Gaussian input at the transmitter and employ maximal ratio combining at the receiver. Let $\mathbf{b}$ be any unit norm vector used for receive combining, then the achievable SNR is given by

$$
\mathrm{SNR}_{1}=\frac{\left|\mathbf{b}^{\dagger} \mathbf{H}^{\dagger} \mathbf{D g}\right|^{2} P}{1+\operatorname{Tr}\left(\mathbf{D}^{\dagger} \mathbf{H} \mathbf{b} \mathbf{b}^{\dagger} \mathbf{H}^{\dagger} \mathbf{D}\right)}
$$

and $\mathbf{D}$ should conform to the power constraint which is given by $\operatorname{Tr}\left(\mathbf{D}\left(\mathbf{g g}^{\dagger} P+\mathbf{I}\right) \mathbf{D}^{\dagger}\right)=P_{R}$. Absorbing the power constraint into the SNR expression we have

$$
\mathrm{SNR}_{1}=\frac{\left|\mathbf{b}^{\dagger} \mathbf{H}^{\dagger} \mathbf{D g}\right|^{2} P P_{R}}{\operatorname{Tr}\left(\mathbf{D}\left(\mathbf{g g}^{\dagger} P+\mathbf{I}\right) \mathbf{D}^{\dagger}\right)+P_{R} \operatorname{Tr}\left(\mathbf{D}^{\dagger} \mathbf{H b b}^{\dagger} \mathbf{H}^{\dagger} \mathbf{D}\right)} .
$$

The capacity of this system is

$$
C_{1}=\max _{\mathbf{b}:\|\mathbf{b}\|=\mathbf{1}} \log \left(1+\mathrm{SNR}_{1}\right)
$$

For the dual MISO network, we construct an achievable scheme that achieves the capacity in (6). Let the transmitted symbol be $\mathbf{x}=\mathbf{b} x^{\prime}$ where $\mathcal{E}\left[\left|x^{\prime}\right|^{2}\right]=P_{R}$. Let $\kappa \mathbf{D}^{\dagger}$ be the relay amplification factor where

$$
\kappa^{2}=\frac{P}{\operatorname{Tr}\left(\mathbf{D}^{\dagger}\left(\mathbf{H b b}^{\dagger} \mathbf{H}^{\dagger} P_{R}+\mathbf{I}\right) \mathbf{D}\right)} .
$$

Here $\kappa$ ensures that the total relay power is $P$. The achievable SNR for this scheme is

$$
\mathrm{SNR}_{2}=\frac{\kappa^{2}\left|\mathbf{g}^{\dagger} \mathbf{D}^{\dagger} \mathbf{H} \mathbf{b}\right|^{2} P_{R}}{1+\kappa^{2} \operatorname{Tr}\left(\mathbf{D g g} \mathbf{D}^{\dagger} \mathbf{D}^{\dagger}\right)}
$$

The achievable SNR after substituting $\kappa$ is given by

$$
\begin{aligned}
\mathrm{SNR}_{2} & =\frac{\left|\mathbf{g}^{\dagger} \mathbf{D}^{\dagger} \mathbf{H b}\right|^{2} P P_{R}}{\operatorname{Tr}\left(\mathbf{D}^{\dagger}\left(\mathbf{H b b}^{\dagger} \mathbf{H}^{\dagger} P_{R}+\mathbf{I}\right) \mathbf{D}\right)+P \operatorname{Tr}\left(\mathbf{D g g}{ }^{\dagger} \mathbf{D}^{\dagger}\right)} \\
& =\mathrm{SNR}_{1} .
\end{aligned}
$$

Thus the MISO relay network can achieve the capacity of the SIMO relay network with a beamforming vector equal to the receive combining vector in the SIMO case.

\section{B. Part $2(M I S O \leq S I M O)$}

Consider the MISO case with source transmit power $P_{R}$ and total relay power $P$. Let $\mathbf{D}$ be the relaying matrix. The capacity of this system is the result of the following optimization problem.

$$
C=\max _{\mathbf{Q}_{\mathbf{x}}, \mathbf{D}} I(\mathbf{x} ; y)
$$

$$
\text { s.t. } \operatorname{Tr}\left(\mathbf{Q}_{\mathbf{x}}\right)=P_{R} \quad \text { and } \quad \operatorname{Tr}\left(\mathbf{D}^{\dagger}\left(\mathbf{H Q}_{\mathbf{x}} \mathbf{H}^{\dagger}+\mathbf{I}\right) \mathbf{D}\right)=P \text {. }
$$

For a given D, Gaussian inputs are optimal and the capacity achieving strategy is beamforming. However there is a power constraint on $\mathbf{D}$ that depends on the input covariance matrix $Q_{x}$. Therefore it is not clear whether beamforming is optimal or not. Furthermore it is not clear whether Gaussian inputs are optimal.

Notice that the relay power constraint depends only on the input covariance matrix and not on the input distribution. For any $\left(\mathbf{D}, \mathbf{Q}_{\mathbf{x}}\right)$ pair that satisfies the power constraints, the best input distribution is Gaussian as it maximizes the mutual information. Let $\left(\mathbf{D}^{\star}, \mathbf{Q}_{\mathbf{x}}^{\star}\right)$ be the optimal pair. Now consider 


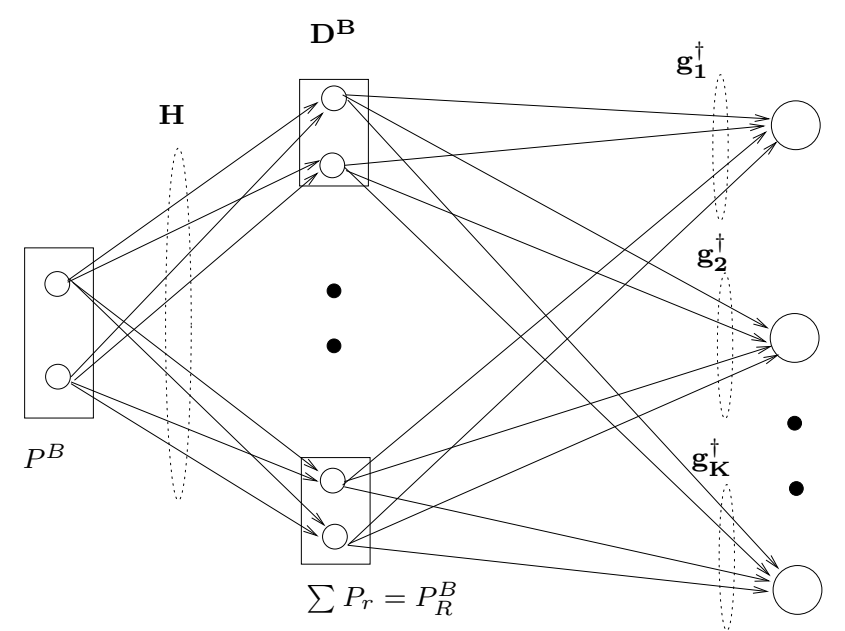

Fig. 2. MIMO broadcast AF relay network

the eigenvalue decomposition of $\mathbf{Q}_{\mathbf{x}}^{\star}$

$$
\mathbf{Q}_{\mathbf{x}}^{\star}=\mathbf{U} \boldsymbol{\Lambda} \mathbf{U}^{\dagger} \Rightarrow \mathbf{x}=\sum_{i=1}^{M} \sqrt{\lambda_{i}} \mathbf{u}_{i} x_{i}
$$

where $\sum_{i=1}^{M} \lambda_{i}=P_{R}$ and $\mathbf{u}_{i}$ is the $i$ th column of $\mathbf{U}$.

We can view the multi-antenna transmitter as $M$ independent sources each with power $\lambda_{i}$. The resultant MAC achieves the same capacity as in (8). In the MAC, the channel from the $i$ th source to the relays is $\mathbf{H u}_{i}$. This MAC relay channel subscribes to the framework in [3]. This channel is dual to the broadcast channel which in turn is inferior to the SIMO relay channel. Thus the SIMO relay network is at least as capable as the MISO relay network.

Discussion: Let us understand why (2) and (4) take different form making it hard to spot a duality connection. For the SIMO channel, let $\mathbf{D}$ be any relay amplification vector that satisfies the relay power constraint. Then the optimal receive combining vector (MRC) is given by

$$
\mathbf{b}^{\star}=\frac{\left(\mathbf{H}^{\dagger} \mathbf{D} \mathbf{D}^{\dagger} \mathbf{H}+\mathbf{I}\right)^{\frac{-1}{2}} \mathbf{H}^{\dagger} \mathbf{D g}}{\left\|\left(\mathbf{H}^{\dagger} \mathbf{D} \mathbf{D}^{\dagger} \mathbf{H}+\mathbf{I}\right)^{\frac{-1}{2}} \mathbf{H}^{\dagger} \mathbf{D g}\right\|} .
$$

From Theorem 1, this vector is also optimal in the MISO relay channel. It is important to note that the effective channel in the MISO channel is not in the same direction as that of the optimal precoding vector. This is because any vector that is parallel to the effective channel will affect the relay power constraint. And the optimum precoding vector need not be along the direction of the effective channel. This explains the difference in the direction of the channel in (4) and (2). This also suggests that the duality is not mainly due to use of the scaled versions of $\mathbf{D}$ but it is rather due to the relay power constraint that couples the two channels.

\section{DUALITY IN MULTI-USER RELAY NETWORKS}

Consider a MIMO broadcast channel with AF relays as shown in Fig. 2. The channel from the transmitter to the relays is denoted by $\mathbf{H}$ while the row vector $\mathbf{g}_{i}^{\dagger}$ represents the channel from the set of relays to the single antenna user $i$. Let the

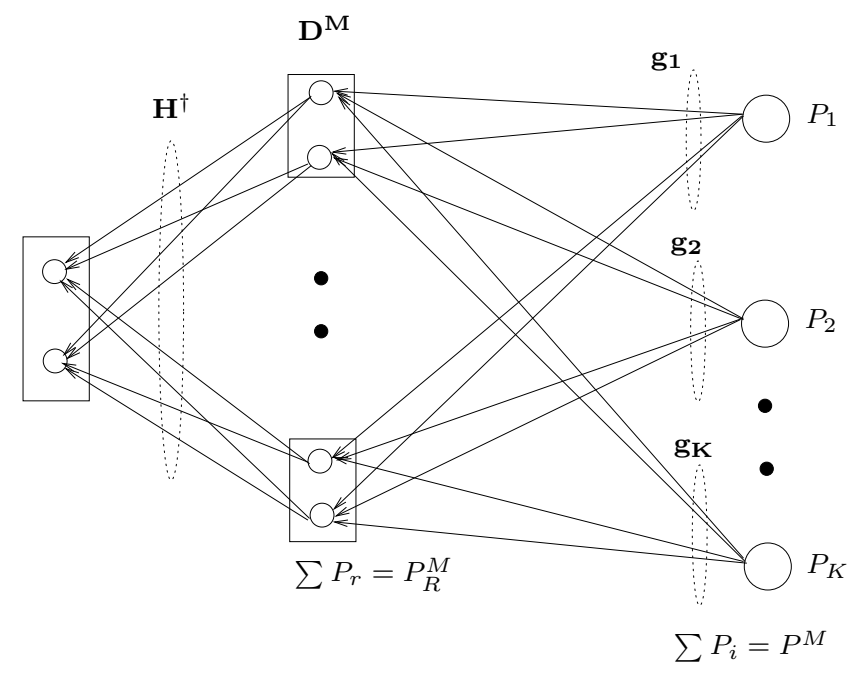

Fig. 3. MIMO multiple access AF relay network

source transmit power be $P^{B}$. The relay amplification matrix employed in this broadcast channel is $\mathbf{D}^{\mathbf{B}}$. The received signal at the $k$ th user is given by

$$
y_{k}=\mathbf{g}_{k}^{\dagger} \mathbf{D}^{\mathbf{B}} \mathbf{H} \mathbf{x}+\mathbf{g}_{k}^{\dagger} \mathbf{D}^{\mathbf{B}} \mathbf{n}_{\mathbf{R}}+n_{k},
$$

where $n_{k}$ and the components of $n_{R}$ are i.i.d. $\mathcal{C N}(0,1)$. The total power consumed by the relays can be calculated as

$$
P_{R}^{B}=\operatorname{Tr}\left(\mathbf{D}^{\mathbf{B}}\left(\mathbf{H} \mathbf{Q}_{\mathbf{x}} \mathbf{H}^{\dagger}+\mathbf{I}\right) \mathbf{D}^{\mathbf{B}^{\dagger}}\right) .
$$

The total power expended across the network is $P_{T}^{B}=P^{B}+$ $P_{R}^{B}$. Now consider the multiple access channel as shown in Fig. 3 obtained from reversing the direction of communication in the broadcast channel. Let $\mathbf{D}^{\mathbf{M}}$ be the relay amplification matrix employed in this channel. The received signal at the base station can be expressed as

$$
\mathbf{y}=\mathbf{H}^{\dagger} \mathbf{D}^{\mathbf{M}} \sum_{k=1}^{K} \mathbf{g}_{k} x_{k}+\mathbf{H}^{\dagger} \mathbf{D}^{\mathbf{M}} \mathbf{n}_{\mathbf{R}}+\mathbf{n}
$$

where $x_{k}$ is the signal transmitted by user $k$ with power $\mathcal{E}\left[\left.|| x_{k}\right|^{2}\right]=P_{k}$. The total power utilized by the relays is

$$
P_{R}^{M}=\operatorname{Tr}\left(\mathbf{D}^{\mathbf{M}}\left(\sum_{k=1}^{K} \mathbf{g}_{k} \mathbf{g}_{k}^{\dagger} P_{k}+\mathbf{I}\right) \mathbf{D}^{\mathbf{M}^{\dagger}}\right)
$$

and the total power expended by the network is $P_{T}^{M}=P_{R}^{M}+$ $P^{M}$ where $P^{M}=\sum_{k=1}^{K} P_{k}$. The following theorem reveals the duality relationship between the relay MAC and and its reciprocal $\mathrm{BC}$ for fixed relaying matrices.

Theorem 2: Consider a $K$ user MIMO AF relay network. Let $\mathbf{D}$ be any relaying matrix in the BC. For any $c \in \mathbb{R}_{+}$, let $c \mathbf{D}^{\dagger}$ be the relay amplification matrix in the reciprocal MAC. Then the following statements are true.

1) MAC-BC duality in MIMO relay networks holds when the total source and relay power are switched in the dual network, i.e. $P_{R}^{M}=P^{B}, P_{R}^{B}=P^{M}$ and

$$
c^{2}=\frac{P_{R}^{M}}{\operatorname{Tr}\left(\mathbf{D}^{\dagger}\left(\sum_{j=1}^{K} \mathbf{g}_{j} \mathbf{g}_{j}^{\dagger} P_{j}+\mathbf{I}\right) \mathbf{D}\right)} .
$$


2) Under a total network power constraint, MAC-BC duality holds when $\mathbf{D}$ and $\mathbf{D}^{\dagger}$ are the relaying matrices used in the MAC and BC respectively. In other words, MAC-BC duality holds when

$$
c=1, \quad P_{R}^{M}+P^{M}=P_{R}^{B}+P^{B} .
$$

3) The values for $c$ given by (13) and (14) are the only cases where MAC-BC duality holds for a total network power constraint.

Proof: For any power allocation $\alpha_{i}$ and precoding vector $\mathbf{u}_{i}$ for user $i$ and for a given encoding order in BC, consider a MAC that uses a decoding order that is the reverse of the encoding order and employs $\mathbf{u}_{i}^{\dagger}$ as the receive combining vector for decoding user $i$ 's signal. The goal is to find a user power allocation strategy in the MAC that would result in achieving the same rate tuple as in the $\mathrm{BC}$. The power allocation for user $i$ will be

$P_{i}=\alpha_{i} \frac{\sum_{j=1}^{i-1}\left|\mathbf{u}_{i}^{\dagger} \mathbf{H}^{\dagger} \mathbf{D}^{\dagger} \mathbf{g}_{j}\right|^{2} P_{j}+\operatorname{Tr}\left(\mathbf{D H} \mathbf{u}_{i} \mathbf{u}_{i}^{\dagger} \mathbf{H}^{\dagger} \mathbf{D}^{\dagger}\right)+\frac{1}{c^{2}}}{\sum_{j=i+1}^{K}\left|\mathbf{g}_{i}^{\dagger} \mathbf{D} \mathbf{H} \mathbf{u}_{j}\right|^{2} \alpha_{j}+\operatorname{Tr}\left(\mathbf{D}^{\dagger} \mathbf{g}_{i} \mathbf{g}_{i}^{\dagger} \mathbf{D}\right)+1}$.

After calculating the total network power in the MAC and $\mathrm{BC}$, the difference in the total network consumption can be obtained as

$$
\Delta P=P_{T}^{M}-P_{T}^{B}=\left(c^{2}-1\right)\left(P_{R}^{B}-P^{M}\right) .
$$

Refer to the Appendix for the derivation. For MAC-BC duality to hold under a total network power constraint, we need to find $c$ such that $\Delta P=0$. From (15), it is clear that the only solutions are

- $c=1$

- $P_{R}^{B}=P^{M}$ which leads to $P_{R}^{M}=P^{B}$ due to equal network power. The value of $c$ can be found from the relay power constraint in the MAC which is given by

$$
c^{2}=\frac{P_{R}^{M}}{\operatorname{Tr}\left(\mathbf{D}^{\dagger}\left(\sum_{j=1}^{K} \mathbf{g}_{j} \mathbf{g}_{j}^{\dagger} P_{j}+\mathbf{I}\right) \mathbf{D}\right)} .
$$

This concludes the proof.

\section{A. Extensions}

1) Linear processing: Theorem 2 holds even when the user signals are encoded and decoded independently at the transmitter and the receiver respectively, i.e., without successive encoding (dirty paper coding) or successive interference cancelation.

2) Multi-antenna users: Theorem 2 also holds when the users have multiple antennas. Using eigenvalue decomposition of the user covariance matrix, the multiple antennas can be reduced to independent single antenna nodes. Consequently the duality result in Theorem 2 directly holds.

Discussion: We stress that the duality result in Theorem 2 holds only for fixed relaying matrices in the MAC and BC. When the relay amplification matrix is fixed in the $B C$, the network reduces to a single hop Gaussian MIMO broadcast channel for which the optimality of dirty paper coding is well known. However, for the joint optimization of the source covariance matrix $\mathbf{Q}_{\mathbf{x}}$ and the relaying matrix $\mathbf{D}$, it is not clear whether dirty paper coding is optimal. For example, consider the MISO relay network in Section II. It can be noticed that the joint optimization of $\mathbf{Q}_{\mathbf{x}}$ and $\mathbf{D}$ resulted in a precoding vector that is not parallel to the effective channel direction. Similarly, in the BC the relay power constraint may affect the choice of the covariance matrix at the transmitter. Since it is not known whether DPC is optimal for any transmit covariance matrix in the classical MIMO broadcast channel (single hop), the optimality of DPC in the MIMO relay network cannot be claimed. To summarize, Theorem 2 suggests that the capacity region of the MAC is equal to the dirty paper coding region of the BC.

For the special case of single antenna source in the $\mathrm{BC}$ with multi-antenna users and relays, it can be established that the DPC rate region is equal to the capacity region of the broadcast relay network.

Theorem 3: Consider a broadcast AF relay channel that has a single antenna transmitter and with the source and relay power constraints $P$ and $P_{R}$ respectively. Its capacity region is equal to the capacity region of the MAC obtained from reversing the communication direction and switching the power constraints, i.e., in the MAC, the sum of user powers is $P_{R}$ while the total relay power constraint is $P$.

Proof Outline: Let us first consider the broadcast relay network. As the transmitter contains only a single antenna, the relay power constraint depends only on $P$ and not on the transmit strategy. Therefore Gaussian inputs are optimal and DPC is the optimal transmit strategy. Choose any $\mathbf{D}$ that satisfies the power constraint and fix any encoding order for the users. Let $\mathbf{b}_{i}^{\dagger}$ be the unit-norm receive combining vector at user $i$. The achievable rates can the be calculated. The capacity region is obtained by optimizing over $\mathbf{b}_{i}$ and $\mathbf{D}$. Now in the MAC, user $i$ employs the beamforming vector $\mathbf{b}_{i}$. Let the decoding order at the destination be opposite to the BC encoding order. Now it can be shown that there exists user power allocation such that the rate tuple in the $\mathrm{BC}$ is achievable in the MAC with total user power $P_{R}$ and sum relay power $P$. This establishes that the MAC is as capable as the $\mathrm{BC}$. The converse is similar to the second part of the proof for Theorem 1. First Gaussian inputs are optimal at each user as the relay power constraint depends only on the user covariance matrix. Then for any covariance matrix, the multiple antenna user can be reduced to many single antenna users. The reduced network resembles a MAC relay network with single antenna sources and destination. For this network, the dual BC is at least as capable.

\section{CONCLUSION}

In this work, we showed that the duality relationships in AF relay networks, that were known for single antenna nodes, hold even when the nodes have multiple antennas. Similar to [3], the dual channel uses a scaled and conjugate-transposed version of the relaying matrix used in the original channel. In addition the source and relay powers are switched so that each hop has the same transmit power in both the channels. In another version of duality, the same relaying matrix is used in 
both the channels. Here the total network power expenditure in the same in the MAC and BC. It is shown in this work that such a duality also holds for MIMO networks. Since both the duality results use a scaled form of the relaying matrix in the channels, an interesting question arises: How many possible duality relationships can be found by using different scaled versions of the relaying matrix in the dual channel? We showed that duality under total network power constraint holds only for the above two cases.

The duality result is valid only when the relaying matrix is fixed. For the case of joint source and relay optimization, we showed that the capacity region of the MAC is equal to the dirty paper coding region of the BC. The optimality of DPC in the broadcast relay network is not established.

\section{APPENDIX}

\section{A. Proof of Theorem 2}

The source in the broadcast channel transmits $K$ independent messages. Let $\mathbf{D}^{\mathbf{B}}=\mathbf{D}$ be the relay amplification matrix. For a fixed $\mathbf{D}$, the network reduces to a single hop MIMO broadcast channel whose capacity region is equal to the dirty paper coding region. Without loss of generality, let user 1 be encoded first, user 2 next and so on. Let $\alpha_{i}$ be the power allocated for the $i$ th user such that $\sum \alpha_{i}=P^{B}$. Let $\mathbf{u}_{i}$ denote an arbitrary unit norm precoding vector at the source for user $i$. Due to successive encoding, the received signal at user $i$ will be

$$
y_{i}=\mathbf{g}_{i}^{\dagger} \mathbf{D} \mathbf{H} \mathbf{u}_{i} x_{i}+\mathbf{g}_{i}^{\dagger} \mathbf{D H} \sum_{j=i+1}^{K} \mathbf{u}_{j} x_{j}+\mathbf{g}_{i}^{\dagger} \mathbf{D} \mathbf{n}_{\mathbf{R}}+n_{i}
$$

The achievable SNR for user $i$ is

$$
\mathrm{SNRB}_{i}=\frac{\left|\mathbf{g}_{i}^{\dagger} \mathbf{D} \mathbf{H} \mathbf{u}_{i}\right|^{2} \alpha_{i}}{\sum_{j=i+1}^{K}\left|\mathbf{g}_{i}^{\dagger} \mathbf{D H} \mathbf{u}_{j}\right|^{2} \alpha_{j}+\operatorname{Tr}\left(\mathbf{D}^{\dagger} \mathbf{g}_{i} \mathbf{g}_{i}^{\dagger} \mathbf{D}\right)+1}
$$

The total power utilized by the relays is

$$
P_{R}^{B}=\operatorname{Tr}\left(\mathbf{D H}\left(\sum_{i=1}^{K} \mathbf{u}_{i} \mathbf{u}_{i}^{\dagger} \alpha_{i}\right) \mathbf{H}^{\dagger} \mathbf{D}^{\dagger}\right) .
$$

Now consider a MAC that is the flipped version of the BC. Let $\mathbf{D}^{\mathbf{M}}=c \mathbf{D}^{\dagger}$ be the relay amplification matrix where $c$ is any real scalar. We choose a decoding order that is reverse to the encoding order in BC, i.e., user 1 is decoded last. Let $\mathbf{u}_{i}^{\dagger}$ be the receive combining vector for user $i$. The received signal at the destination after canceling the interference of sources $K$ to $i+1$ is

$$
\mathbf{y}=c \sum_{j=1}^{i} \mathbf{H}^{\dagger} \mathbf{D}^{\dagger} \mathbf{g}_{j} x_{j}+c \mathbf{H}^{\dagger} \mathbf{D}^{\dagger} \mathbf{n}_{\mathbf{R}}+\mathbf{n} .
$$

In this channel, the achievable SNR for user $i$ is

$$
\mathrm{SNRM}_{i}=\frac{c^{2}\left|\mathbf{u}_{i}^{\dagger} \mathbf{H}^{\dagger} \mathbf{D}^{\dagger} \mathbf{g}_{i}\right|^{2} P_{i}}{c^{2} \sum_{j=1}^{i-1}\left|\mathbf{u}_{i}^{\dagger} \mathbf{H}^{\dagger} \mathbf{D}^{\dagger} \mathbf{g}_{j}\right|^{2} P_{j}+c^{2} \operatorname{Tr}\left(\mathbf{D H} \mathbf{u}_{i} \mathbf{u}_{i}^{\dagger} \mathbf{H}^{\dagger} \mathbf{D}^{\dagger}\right)+1} .
$$

The total power utilized by the relays in the MAC is

$$
P_{R}^{M}=c^{2} \operatorname{Tr}\left(\mathbf{D}^{\dagger}\left(\sum_{j=1}^{K} \mathbf{g}_{j} \mathbf{g}_{j}^{\dagger} P_{j}+\mathbf{I}\right) \mathbf{D}\right) .
$$

Now we are interested in finding the power allocation to users in the MAC such that the rate tuple in the BC is achieved in the MAC. The total network power expended in the MAC reduces to

$$
\begin{aligned}
P_{T}^{M}=c^{2} \sum_{j=1}^{K} P_{j}(1+ & \left.\operatorname{Tr}\left(\mathbf{D}^{\dagger} \mathbf{g}_{j} \mathbf{g}_{j}^{\dagger} \mathbf{D}\right)\right)+c^{2} \operatorname{Tr}\left(\mathbf{D D}^{\dagger}\right) \\
& +\left(1-c^{2}\right) \sum_{j=1}^{K} P_{j}
\end{aligned}
$$

Equating (16) and (17), and using the relation

$$
\sum_{i=1}^{K} \sum_{j=1}^{i-1}\left|\mathbf{u}_{i}^{\dagger} \mathbf{H}^{\dagger} \mathbf{D}^{\dagger} \mathbf{g}_{j}\right|^{2} P_{j} \alpha_{i}=\sum_{i=1}^{K} \sum_{j=i+1}^{K}\left|\mathbf{g}_{i}^{\dagger} \mathbf{D H} \mathbf{u}_{i}\right|^{2} \alpha_{j} P_{i}
$$

the total network power in the MAC can be obtained as

$$
\begin{aligned}
P_{T}^{M}=\sum_{i=1}^{K} \alpha_{i}(1 & \left.+c^{2} \operatorname{Tr}\left(\mathbf{D H} \mathbf{u}_{i} \mathbf{u}_{i}^{\dagger} \mathbf{H}^{\dagger} \mathbf{D}^{\dagger}\right)\right) \\
& +\left(1-c^{2}\right) \sum_{j=1}^{K} P_{j}+c^{2} \operatorname{Tr}\left(\mathbf{D}^{\dagger} \mathbf{D}\right) .
\end{aligned}
$$

For the broadcast channel, the total power spent across the network is

$$
P_{T}^{B}=\sum_{i=1}^{K} \alpha_{i}+\operatorname{Tr}\left(\mathbf{D}\left(\mathbf{I}+\sum_{i=1}^{K} \mathbf{H} \mathbf{u}_{i} \mathbf{u}_{i}^{\dagger} \mathbf{H}^{\dagger} \alpha_{i}\right) \mathbf{D}^{\dagger}\right) .
$$

To achieve the same rate tuple in both the BC and MAC, the difference in the total network power when $\mathbf{D}$ and $c \mathbf{D}^{\dagger}$ are the relaying matrices for the $\mathrm{BC}$ and MAC respectively is

$$
\begin{aligned}
\Delta P & =P_{T}^{M}-P_{T}^{B} \\
& =\left(c^{2}-1\right)\left(\operatorname{Tr}\left(\mathbf{D}\left(\mathbf{I}+\sum_{i=1}^{K} \mathbf{H} \mathbf{u}_{i} \mathbf{u}_{i}^{\dagger} \mathbf{H}^{\dagger} \alpha_{i}\right) \mathbf{D}^{\dagger}\right)-P^{M}\right) \\
& =\left(c^{2}-1\right)\left(P_{R}^{B}-P^{M}\right) .
\end{aligned}
$$

Similarly, if we start with a MAC that uses D and find the power allocation for a dual $\mathrm{BC}$ that employs $c \mathbf{D}^{\dagger}$, it can be shown that $\Delta P=P_{T}^{B}-P_{T}^{M}=\left(c^{2}-1\right)\left(P_{R}^{M}-P^{B}\right)$.

\section{REFERENCES}

[1] S. Vishwanath, N. Jindal, A. J. Goldsmith, "Duality, achievable rates and sum-rate capacity of Gaussian MIMO broadcast channel," IEEE Transactions on Information Theory, vol. 49, pp. 2658-2668, 2003.

[2] P. Viswanath and D. Tse, "Sum Capacity of the Multiple Antenna Gaussian Broadcast Channel and Uplink-Downlink Duality," IEEE Transactions on Information Theory, vol. 49, no. 8, pp. 1912-1923, Aug 2003.

[3] S. A. Jafar, K. Gomadam, C. Huang, "Duality and Rate Optimization for Multiple Access and Broadcast Channels With Amplify-and-Forward Relays," IEEE Transactions on Information Theory, vol. 53, pp. 33503370, October 2007.

[4] K. Gomadam, S. Jafar, "The effect of noise correlation in AF relay networks," http://arxiv.org/abs/0707.1064v1.

[5] A. Coso, C. Ibars, "The Amplify-based Multiple-Relay Multiple-Access Channel: Capacity Region and MAC-BC Duality," in IEEE ITW, 2007. 